\title{
The Need is Growing up for a New Replacing Biointegrated Material
}

\section{Laith Mahmoud Abdulhadi*}

Faculty of Dentistry, MAHSA University, Kuala Lumpur, Malaysia

Anaplastology is the interaction of art, science and technology for prosthetic rehabilitation of an absent, disfigured, or malformed part of the human body. In Anaplastology, the objective is to replace the mutilated structure by either compatible living tissue or artificial biocompatible material. The preference to use which mode is keyed to many host, technical and facility factors. Generally, artificial replacement is indicated in small as well as large defects and nearly in any place of the body and at affordable cost. One of the advantages of the medical-grade silicone (MG-S) is the possible color matching to some extent of the defect tissue or its environment plus its texture. However, the silicone still needs much improvement to retain the initial physical and mechanical properties for acceptable longevity after use and to resist the aging process associated with all types of fabricated material.

The biocompatibility of silicone for external use is considered more acceptable than the implantable one. However, some type of allergic reaction may be encountered from time to time in some allergic patients and the material cannot be used even for external purposes.

Epithesis is the replacement of missing soft tissue alone or combined with bonny substructures and located extra orally. It is mainly fabricated of medical grade silicone and sometimes supported or integrated with hard base like acrylic resin. The replacement of facial or body missing structures normally performed using MG-silicone. This material with all its brands are generally grouped into three categories: non implantable, short-term implantable, and long-term implantable. Materials approved as USP2 Class V and VI can be considered medical grade. The problems encountered in using MG-S are multiple and many pages may be filled. The manipulation is surely simple but color matching is still at the middle of the tunnel and it is not easy for the practitioner to produce live color and texture even with the introduction of many coloring systems and techniques to the profession to overcome the deficiencies. The physical properties of the material still hold much weakness like tearing resistance when it is used in thin layer especially at the margin of the defect or the opening structures. Generally, the retentive means used to retain the epithesis mainly depend on using implants-anchor, biologic adhesive or other simple extra means. Using implants for fixing the prosthesis has some advantages over many disadvantages especially for the patient. Biologic adhesive is considered an auxiliary mean and should be used in conjugation with another retentive method. It is indicated in case of light-weight small epithesis only. Therefore, many researches are needed to enhance the physical, mechanical, adherence and biocompatibility of the silicone material or to develop new brand that fulfill the updated requirements and at the same time more simplified automatic color matching technology so that it will be affordable to all new and experienced practitioners as well at a cost-effective manner. In the market, there are new systems marketed as a matching color effective however; no research work supports the manufacturer's claim.

Another opinion may arise regarding the future of replacing method and material. Generally, the best way for reconstruction of mutilation is to use biologically integrated materials. The methodology and the materials should be revised also in the light of the new advances in the biointegration.

To make these visions and suggestions applicable and possible, the development should include the training programs as well as new big research funds should be addressed.

The training programs which are offered by some well-known organizations and academic institutes seems to be very efficient currently for anaplastologists. However, with the development of advanced bioengineering and tissue culturing, the program should be more advanced and updated to cope with these visions.

In the meantime, the biocompatibility of the silicone material should be questioned and biologic integration should enter this discipline as well. The introduction of midface replacement as a method to substitute or correct disfigured midface seems a good solution but with many arguments regarding its success rate, ethical issues, eases of performance, host immune reaction, find compatible donor and at appropriate cost. Therefore, another management method should be addressed.

The future of reconstruction and management of postsurgical or posttraumatic defects is mainly dependent on the development of new materials, biointegration and new replacing surgery.

As a conclusion, the anaplastology with its two integrated subspecialty method of management; surgical and prosthetic is at the midway position between many therapeutic options to be selected for further development to resolve the critical situations and offering better results at affordable cost and simple technique that can be used by even newly graduated practitioners. The interested researchers and developing companies are invited to investigate and fabricate new brands of materials with detailed enhanced features and properties to fulfill the requested objectives.
*Corresponding author: Laith Mahmoud Abdulhadi, Professor, Faculty of Dentistry, MAHSA University, Kuala Lumpur, Malaysia, E-mail: laithmahmoud@mahsa.edu.my

Received Januay 08, 2013; Accepted Januay 08, 2013; Published Januay 10 2013

Citation: Abdulhadi LM (2013) The Need is Growing up for a New Replacing Biointegrated Material. Anaplastology 2: e108. doi: 10.4172/2161-1173.1000e108

Copyright: (c) 2013 Abdulhadi LM, et al. This is an open-access article distributed under the terms of the Creative Commons Attribution License, which permits unrestricted use, distribution, and reproduction in any medium, provided the original author and source are credited. 